\title{
Broadband $\mathrm{CO}_{2}$ measurements with a VIPA spectrometer in the near-infrared
}

\author{
Grzegorz Kowzan, ${ }^{*}$ Magdalena Paradowska, Mikołaj Zaborowski, Mateusz Borkowski, Piotr Ablewski, Szymon \\ Wójtewicz, Kamila Stec, Tadeusz Robaczewski, Daniel Lisak, Ryszard S. Trawiński, Piotr Masłowski \\ Institute of Physics, Faculty of Physics, Astronomy and Informatics, Nicolaus Copernicus University in Torun, \\ Grudziadzka 5, 87-100 Torun, Poland
}

Received September 21, 2015; accepted September 29, 2015; published September 30, 2015

\begin{abstract}
We demonstrate near-infrared cavity-enhanced optical frequency comb spectroscopy of $\mathrm{R}$ branch of $\mathrm{CO}_{2}$ overtone transitions around $1.57 \mu \mathrm{m}$. The measurement setup is based on an Er:fiber optical frequency comb, high finesse cavity and a VIPA spectrometer.A dither locking scheme provides robust operation and high absorption sensitivity enhancement, while a VIPA etalon-based spectrometer provides rapid broadband acquisition with $600 \mathrm{MHz}$ spectral resolution. The sensitivity of the system reaches $2.3 \times 10^{-9} \mathrm{~cm}^{-1}$ at $2 \times 82 \mathrm{~s}$ acquisition time. We verify the resolution of the experimental setup by comparing the measured spectrum with the high-quality spectrum obtained with a cavity ring-down spectrometer.
\end{abstract}

With the development of optical frequency comb (OFC) at the turn of the millennium the optical absorption spectroscopy obtained a versatile tool for frequency measurements [1-3]. When used directly to interrogate an atomic or molecular sample, the broadband spectrum of optical frequency comb offers high sensitivity in a wide spectral range, with acquisition time of the order of seconds or lower. It removes the need for stepwise tuning of a continuous wave (cw) laser, allowing to sense the absorption of tens of molecular lines simultaneously and minimize the influence of long term drifts.

Multiple approaches have been demonstrated with this technique until now [4-6], including cavity ring-down spectroscopy [7], dual-comb spectroscopy [8-10], cavityenhanced Fourier Transform Spectroscopy [11,12] or noise immune cavity-enhanced optical heterodyne molecular spectroscopy (NICE-OHMS) [13]. In cavityenhanced measurements, the overlap between OFC and cavity modes is ensured by two approaches: tight lock usually performed by two-point Pound-Drever-Hall technique $[11,14]$ and so called 'dither lock', where the cavity length is modulated so that the OFC and cavity resonances periodically coincide $[15,16]$. As shown before [4,17], the first approach offers the highest transmitted power and enhancement of the effective optical length $\left(L_{e f f}=\beta \mathrm{F} / \pi \mathrm{L}\right.$, where $\mathrm{F}$ is the cavity finesse, $\mathrm{L}-$ the cavity length and $\beta$ - an enhancement factor equal to 2 in this case), putting high demands on locking speed. The requirements of the second approach are much lower

*E-mail: gkowzan@fizyka.umk.pl at the price of lower transmission and decreased effective optical length depending on locking settings.

In all mentioned setups multiple approaches were used to disperse an optical frequency comb spectrum [4-6]. Spectrometers consisting of a virtually imaged phase array (VIPA) etalon [18-20], a diffraction grating and a camera are attractive because they provide rapid acquisition, limited only by the camera speed, in a compact package without any moving parts. So far, none of the presented frequency comb VIPA spectrometers have managed to combine cavity-enhanced sensitivity, broad spectral coverage, high signal-to-noise ratio, rapid acquisition and self-referenced frequency axis. This paper presents such a system built out of off-the-shelf components in a simple compact setup.

We report the measurements of the $\mathrm{R}$ branch of a $\mathrm{CO}_{2}$ ro-vibrational spectrum in the $1565 \div 1580 \mathrm{~nm}$ range, important due to its application in carbon cycle monitoring [21,22]. The results of the measurements made by a VIPA-based cavity-enhanced setup with a dither locking scheme [7] are analysed in terms of sensitivity and spectral resolution.

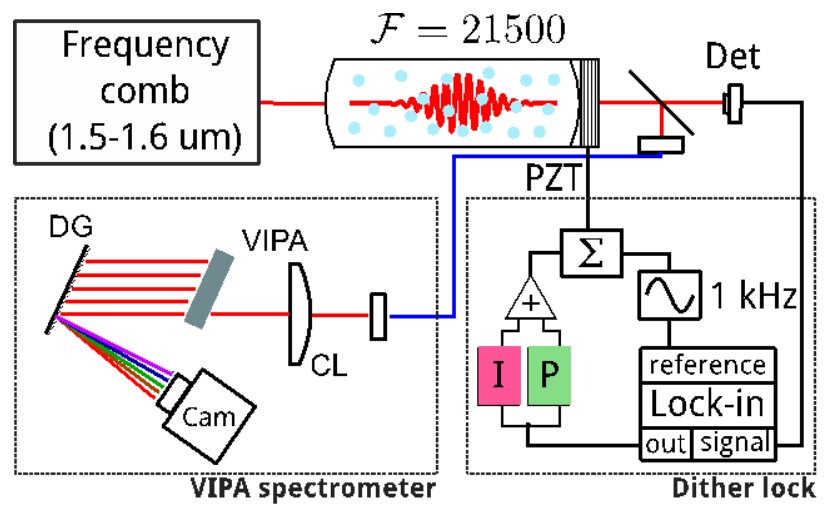

Fig. 1. The scheme of experimental setup. $F$ - cavity finesse, PZT piezeoelectrical transducer, CL - collimating lens, DG - diffraction grating, PI - proportional and integrating servo, Det - InGaAs detector, blue - polarization-maintaining fiber, Cam - InGaAs camera.

Figure 1 outlines the experimental setup. The source of light in the system is Er:fiber optical frequency comb 
(Menlo FC1500) with a repetition rate of $250 \pm 2 \mathrm{MHz}$ and spectrum spanning $1.5 \div 1.6 \mu \mathrm{m}$. The mode-matched comb beam is coupled into a high finesse enhancement cavity (finesse $F=21500$ ). The stable transmission of a comb spectrum through the cavity is achieved with a dither locking scheme: a low amplitude $1 \mathrm{kHz}$ sine wave is applied to the cavity piezotransducer (PZT), modulating the cavity length. This modulation periodically puts cavity modes into resonance with incident comb modes, resulting in periodic peaks in the cavity transmission signal detected by InGaAs detector (Det). This signal is fed to the lock-in amplifier (SR530), referenced to the same signal as the one modulating the cavity length. The phase-sensitive operation of a lock-in amplifier results in an output signal whose sign is dependent on the direction of detuning of cavity length from the resonance condition and provides a good error signal. Subsequently, this error signal is amplified and integrated by a PI controller (NewFocus LB1005) and fed back to the cavity PZT with a feedback bandwidth of approx. $10 \mathrm{~Hz}$. In spite of a low frequency bandwidth of the feedback, this locking scheme provides stable transmission of the whole incident spectrum and maintains hours-long operation. The dispersion system based on a virtually imaged phase array (VIPA) was described extensively in the literature [1820]. Briefly, it consists of a VIPA - a solid etalon with reflective coating optimized for $1.5 \div 1.65 \mu \mathrm{m}$ wavelength range, a $600 / \mathrm{mm}$ reflective grating and an MCT photodetector array. The etalon FSR depends on the tilt from the vertical position and was verified to be $53.3 \mathrm{GHz}$ in the experiment. The system allows to simultaneously acquire approx. $38 \mathrm{~nm}$-wide spectrum of $\sim 15000$ spectral elements with a minimum acquisition time of $2 \times 16 \mathrm{~ms}$. The imaged spectral region can be tuned by simple grating rotation. The VIPA-grating system images a twodimensional diffraction pattern onto the camera (example in Fig. 2a), from which a traditional absorption spectrum can be recovered.
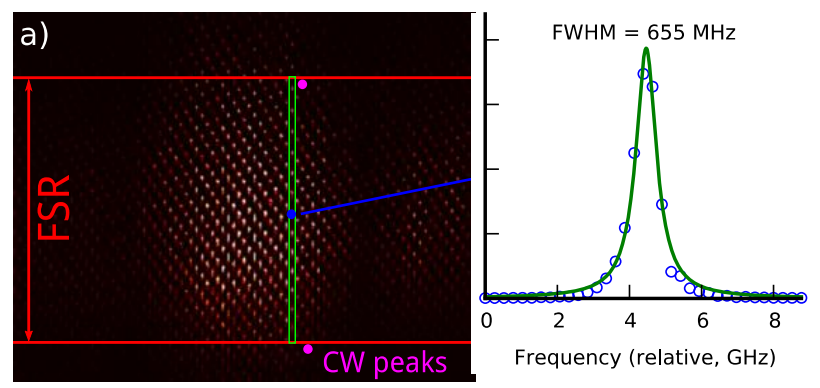

Fig. 2. a) The image from MCT camera with single comb line-resolved spectrum and peaks of cw calibration laser. FSR - VIPA free spectral range b) line shape of single comb line (blue circles) and fit of Lorentzian function (green).

The frequency axis of the final spectrum was calibrated by measurements of comb-resolved spectrum of stabilized frequency comb, shown in Fig. 1a. The spectrum was obtained by introducing mismatch between the cavity FSR and repetition rate of the comb, which results in the comb line spacing equal to approx. $4 \mathrm{GHz}$. The spectrum was then used to establish correspondence between the position on the camera and wavelength with accuracy better than $500 \mathrm{MHz}$. To measure the instrumental line shape (ILS) of our detection system, we have used the image of a single comb-resolved spectrum shown in Fig. $2 \mathrm{a}$ and fitted Lorentzian line shape of a single comb component, as shown in panel $2 \mathrm{~b}$. The obtained shape with a line width of $655 \mathrm{MHz}$ (FWHM) is the instrumental function of the system.

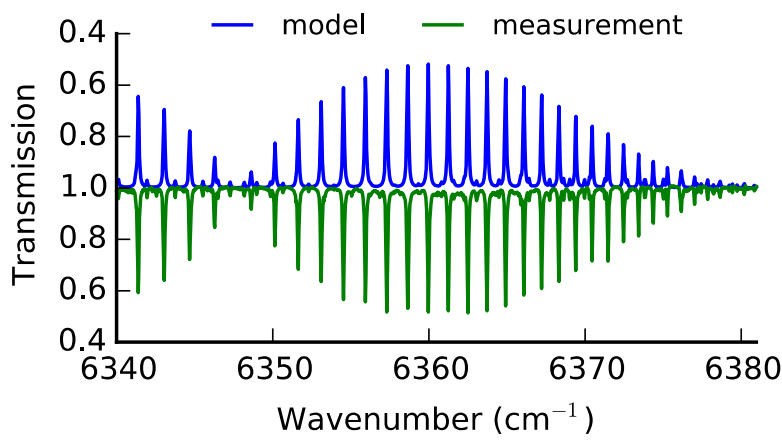

Fig. 3. The spectrum of $\mathrm{CO}_{2} \mathrm{R}$ branch overtone transitions around $6360 \mathrm{~cm}^{-1}$. The sample gas was 300Torr of $2000 \mathrm{ppm} \mathrm{CO}_{2}$ in Ar. Total acquisition time of the spectrum is $2 \times 82 \mathrm{~s}$.

With the described system we have performed measurements of overtone spectrum of $\mathrm{CO}_{2}$ diluted in $\mathrm{Ar}$ at 300 Torr pressure. Figure 3 shows the measured spectrum in the $6340 \div 6380 \mathrm{~cm}^{-1}$ range (green), compared with a fitted model spectrum based on HITRAN database [23] (blue, inverted for clarity). The discrepancy between the measured and modeled line widths is due to the use of broadening coefficients for $\mathrm{CO}_{2}$-air system in the model.

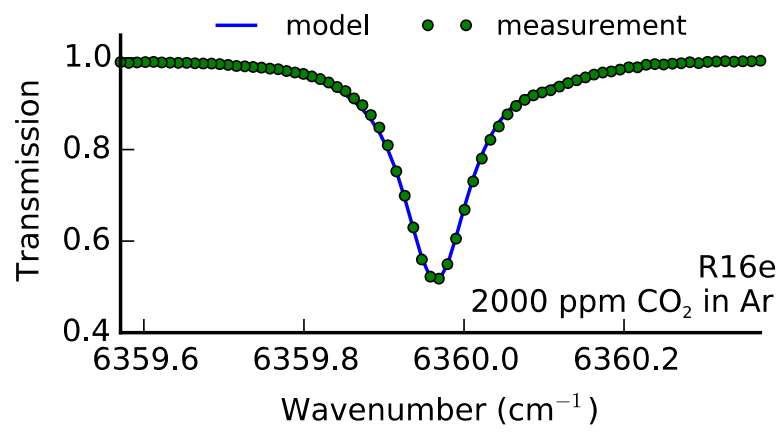

Fig. 4. The measurement of $\mathrm{R} 16 \mathrm{e}$ line of $\mathrm{CO}_{2}$ in $\mathrm{Ar}$ at 300Torr pressure (green full circles) and the fit based on Voigt profile (blue).

To verify the spectral performance of the broadband system we have fitted R16e line with the cavity transmission model [14] assuming the Voigt profile (VP). The line position, line intensity and pressure shift fixed to the reference values were obtained for this line with $\mathrm{cw}$ 
FS-CRDS system available in our laboratory [24, 25]. The fitted value of Lorentzian line width agrees with the sum of the FS-CRDS reference value and instrumental line-shape width (shown in Fig. 2b). The agreement of the fit with the measured spectrum proves the resolving power of the system and the lack of dispersion effects in the line shape, which are present in tight locking schemes. The fitted value of the mixing ratio for $\mathrm{CO}_{2}$ molecules is significantly lower $(780 \mathrm{ppm})$ than the actual value (2000ppm), which is consistent with the observation of decreased effective length in the cavity for a swept locking scheme $[4,5]$ and points to the need of system calibration with a sample of known composition.

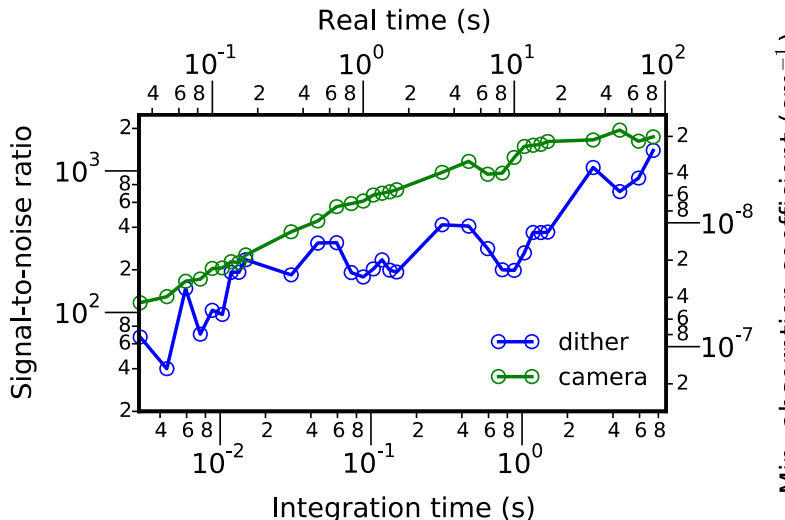

Fig. 5. Signal-to-noise ratio and corresponding minimum detectable absorption coefficient as a function of averaging time. Dither curve corresponds to real measurements, while camera curve is a hypothetical camera noise-limited performance of our system.

We evaluated noise of the system for different averaging times (Fig. 5) by calculating the standard deviation of the center part of normalized spectra [11], i.e. the ratio of two consecutive spectra acquired for specified averaging time. Since the maximum physically meaningful signal value for such a ratio is equal to one, we calculate the signal-to-noise ratio as inverse of standard deviation. By analogy, we obtain an SNR figure for camera noise, using the averaged cavity-transmitted light level as a reference signal value. The minimum detectable absorption coefficient was calculated as a ratio of standard deviation and effective optical path, given by $\beta \mathrm{FL} / \pi$ [4]. In our case we assume the worst-case $\beta=0.73$, as derived from the fit shown in Fig. 4. For our longest acquisition time of $2 \times 7.4 \mathrm{~s}(2 \times 83 \mathrm{~s}$ of real time $)$, we obtain an SNR ratio of 1450 , corresponding to $\alpha_{\min }=2.3 \times 10^{-9} \mathrm{~cm}^{-1}$ and $\alpha_{\min }=3.3 \times 10^{-11} \mathrm{~cm}^{-1}$ per spectral element.

To summarize, a broadband high-resolution fast acquisition spectrometer working in the near-infrared wavelength range was presented. The measurements of $\mathrm{CO}_{2}$ overtone transitions around $6360 \mathrm{~cm}^{-1}$ and comparison with high-quality line-shape parameters show that the system allows for speed and sensitivity, even with a relatively simple and slow cavity-comb locking scheme. This system performance can be increased by the use of a faster, InGaAs-based camera. When using the resolved single comb lines for absorption measurement, the system will be able to deliver an ILS-free spectrum with a frequency axis given by a stabilized frequency comb.

The research is supported by the Foundation for Polish Science HOMING PLUS and TEAM Projects as well as the by Polish National Science Centre Project no. DEC2012/05/D/ST2/01914.

\section{References}

[1] S.A. Diddams, D.J. Jones, J. Ye, S.T. Cundiff, J.L. Hall, J.K. Ranka et al., Phys. Rev. Lett. 84, 5102 (2000).

[2] J.L. Hall, Rev. Mod. Phys. 78, 1279 (2006).

[3] T.W. Hänsch, Rev. Mod. Phys. 78, 1297 (2006).

[4] M.J. Thorpe, J. Ye, Appl. Phys. B 91, 397 (2008).

[5] F. Adler, M.J. Thorpe, K.C. Cossel, J. Ye, Annu. Rev. Anal. Chem. 3, 175 (2010)

[6] P. Masłowski, K.C. Cossel, A. Foltynowicz, J. Ye, Cavity-Enhanced Spectroscopy and Sensing, G. Gagliardi, H.-P. Loock, eds., Springer Series in Optical Sciences (Springer Berlin Heidelberg, 2014), Vol. 179 , p. 271.

[7] M.J. Thorpe, K.D. Moll, R.J. Jones, B. Safdi, J. Ye, Science 311, 1595 (2006).

[8] F. Keilmann, C. Gohle, R. Holzwarth, Opt. Lett. 29, 1542 (2004).

[9] I. Coddington, W.C. Swann, N.R. Newbury, Phys. Rev. Lett. 100, 013902 (2008).

[10] B. Bernhardt, A. Ozawa, P. Jacquet, M. Jacquey, Y. Kobayashi, T. Udem et al., Nat. Photonics 4, 55 (2010).

[11] A. Foltynowicz, T. Ban, P. Masłowski, F. Adler, J. Ye, Phys. Rev. Lett. 107, 233002 (2011).

[12] S. Kassi, K. Didriche, C. Lauzin, X. de G. d'Elseghem Vaernewijckb, A. Rizopoulos, M. Herman, Spectrochim. Acta. A. Mol. Biomol. Spectrosc. 75, 142 (2010)

[13] A. Khodabakhsh, A.C. Johansson, A. Foltynowicz, Appl. Phys. B 119, 87 (2015).

[14] A. Foltynowicz, P. Masłowski, A.J. Fleisher, B.J. Bjork, J. Ye, Appl. Phys. B 110, 163 (2012).

[15] T. Gherman, D. Romanini, Opt. Express 10, 1033 (2002).

[16] M.J. Thorpe, D. Balslev-Clausen, M.S. Kirchner, J. Ye, Opt. Express 16, 2387 (2008).

[17] D. Romanini, I. Ventrillard, G. Mejean, J. Morville, E. Kerstel, CavityEnhanced Spectroscopy and Sensing, G. Gagliardi and H.-P. Loock, eds., Springer Series in Optical Sciences (Springer Berlin Heidelberg, 2014), Vol. 179, p. 1.

[18] S. Xiao, A.M. Weiner, C. Lin, Quantum Electron. IEEE J. Of 40, 420 (2004).

[19] M. Shirasaki, Opt. Lett. 21, 366 (1996).

[20] S. Xiao, A.M. Weiner, Opt. Express 12, 2895 (2004).

[21] P.M. Cox, R.A. Betts, C.D. Jones, S.A. Spall, I.J. Totterdell, Nature 408, 184 (2000).

[22] A. Butz, S. Guerlet, O. Hasekamp, D. Schepers, A. Galli, I. Aben et al., Geophys. Res. Lett. 38, (2011)

[23] L S. Rothman, I.E. Gordon, Y. Babikov, A. Barbe, D. Chris Benner, P.F. Bernath et al., J. Quant. Spectrosc. Radiat. Transf. 130, 4 (2013).

[24] A. Cygan, S. Wójtewicz, J. Domysławska, P. Masłowski, K. Bielska, M. Piwiński et al., Eur. Phys. J. Spec. Top. 222, 2119 (2013).

[25] S. Wójtewicz, K. Stec, P. Masłowski, A. Cygan, D. Lisak, R.S.Trawiński et al., J. Quant. Spectrosc. Radiat. Transf. 130, 191 (2013) 